\title{
The HIF1a/HIF2a-miR210-3p network regulates glioblastoma cell proliferation, dedifferentiation and chemoresistance through EGF under hypoxic conditions
}

\author{
Pan Wang ${ }^{1,2}$, Qian Yan ${ }^{1}$, Bin Liao ${ }^{1}$, Lu Zhao', Shuanglong Xiong ${ }^{3}$, Junwei Wang ${ }^{1}$, Dewei Zou', Jinyu Pan',
} Liangqi Wu ${ }^{4,5}$, Yangmin Deng ${ }^{1}$, Nan $W^{1}$ and Sheng Gong $\mathbb{D}^{1}$

\begin{abstract}
Hypoxia-inducible factor 1a (HIF1a) promotes the malignant progression of glioblastoma under hypoxic conditions, leading to a poor prognosis for patients with glioblastoma; however, none of the therapies targeting HIF1a in glioblastoma have successfully eradicated the tumour. Therefore, we focused on the reason and found that treatments targeting HIF1a and HIF2a simultaneously increased tumour volume, but the combination of HIF1a/HIF2a-targeted therapies with temozolomide (TMZ) reduced tumourigenesis and significantly improved chemosensitization. Moreover, miR-210-3p induced HIF1a expression but inhibited HIF2a expression, suggesting that miR-210-3p regulates HIF1a/HIF2a expression. Epidermal growth factor (EGF) has been shown to upregulate HIF1a expression under hypoxic conditions. However, in the present study, in addition to the signalling pathways mentioned above, the upstream proteins HIF1a and HIF2a have been shown to induce EGF expression by binding to the sequences AGGCGTGG and GGGCGTGG. Briefly, in a hypoxic microenvironment the HIF1a/HIF2a-miR210-3p network promotes the malignant progression of glioblastoma through a positive feedback loop with EGF. Additionally, differentiated glioblastoma cells underwent dedifferentiation to produce glioma stem cells under hypoxic conditions, and simultaneous knockout of HIF1a and HIF2a inhibited cell cycle arrest but promoted proliferation with decreased stemness, promoting glioblastoma cell chemosensitization. In summary, both HIF1a and HIF2a regulate glioblastoma cell proliferation, dedifferentiation and chemoresistance through a specific pathway, which is important for glioblastoma treatments.
\end{abstract}

\section{Introduction}

Glioblastoma (GBM) undergoes malignant progression under hypoxic conditions $s^{1,2}$, which are mainly regulated by HIF $1 \alpha$ and HIF $2 \alpha^{3-6}$. Both HIF1 $\alpha$ and HIF $2 \alpha$ initially regulate the malignant progression of $\mathrm{GBM}^{7}$, but as the

\footnotetext{
Correspondence: Nan Wu (wunan881@tmmu.edu.cn) or

Sheng Gong (Gongsheng@tmmu.edu.cn)

${ }^{1}$ Department of Neurosurgery, Chongqing General Hospital, University of

Chinese Academy of Sciences, 401147 Chongqing, China

${ }^{2}$ Chongqing Medical University, 400016 Chongqing, China

Full list of author information is available at the end of the article

These authors contributed equally: Pan Wang, Qian Yan

Edited by B. Joseph
}

tumour progresses, the effect of HIF2 $\alpha$ on tumour growth decreases, while HIF $1 \alpha$ becomes increasingly important ${ }^{8}$. These results lead to the development of drugs targeting of HIF1 $\alpha$, aiming to inhibit GBM growth in patients ${ }^{9-11}$. Unfortunately, this targeted therapy has not been successful, since it is unable to remarkably reduce the tumour volume. Therefore, we individually or simultaneously knocked out HIF1 $\alpha$ and HIF2 $\alpha$ to determine the reason.

Importantly, hypoxia-related miRNAs have key functions in the malignancy of tumour ${ }^{12,13}$, which exhibit altered expression under hypoxic conditions, thus regulating the malignant progression of $\mathrm{GBM}^{14,15}$. By

\section{(c) The Author(s) 2020}

(c) (i) Open Access This article is licensed under a Creative Commons Attribution 4.0 International License, which permits use, sharing, adaptation, distribution and reproduction c. in any medium or format, as long as you give appropriate credit to the original author(s) and the source, provide a link to the Creative Commons license, and indicate if changes were made. The images or other third party material in this article are included in the article's Creative Commons license, unless indicated otherwise in a credit line to the material. If material is not included in the article's Creative Commons license and your intended use is not permitted by statutory regulation or exceeds the permitted use, you will need to obtain permission directly from the copyright holder. To view a copy of this license, visit http://creativecommons.org/licenses/by/4.0/. 
examining the hypoxia-related miRNAs in glioma, researchers have shown that miR-210-3p may be related to tumour growth through a HIF1 $\alpha$-dependent mechanism $^{16,17}$. However, as shown in our study, a mutual regulatory feedback loop exists between HIF $1 \alpha / \mathrm{HIF} 2 \alpha$ and miR210-3p and subsequently contributes to GBM malignant progression.

Epidermal growth factor (EGF) is expressed at high levels under hypoxic conditions ${ }^{18}$ and regulates GBM growth through EGFR and PI3K/AKT signalling pathways $^{19,20}$. A meaningful observation is that one of the downstream genes of PI3K/AKT signalling pathway is HIF $1 \alpha^{21}$, and many studies have confirmed that HIF1 $\alpha$ expression depends on EGF ${ }^{18,19}$. Therefore, the mechanism of GBM growth under hypoxic conditions defined by previous studies is that EGF present at high levels binds to EGFR, which then activates the PI3K/AKT signalling pathway and induces steady expression of HIF1 $\alpha$ to promote the malignant progression of the tumour ${ }^{19}$. However, few studies have directly assessed the mutual relationship between HIF1 $\alpha / \mathrm{HIF} 2 \alpha$ and EGF, and we identified both HIF1 $\alpha$ and HIF $2 \alpha$ as upstream factors that contribute to regulating EGF by binding to a similar HRE sequence in our study. Therefore, a regulatory mechanism between HIF $1 \alpha / H I F 2 \alpha$ and EGF exists, and the activation of this pathway promotes the malignant progression of GBM.

\section{Materials and methods \\ Public data collection}

Protein expression and correlation, disease-free survival (DFS) and overall survival (OS) were analysed for patients included in TCGA, GTEx and CCLE databases using GEPIA (http://gepia.cancer-pku.cn/detail.php).

\section{Cell isolation and cell culture}

U87MG cells and primary glioblastoma (GBM) cells isolated from tissues after surgery were used in the study, and the detailed methods of sorting GBM cells are presented in the supplementary materials. The tumour tissues obtained from patients were anonymized. U87MG cells were authenticated by STR profiling and all the cells were verified none mycoplasma contamination.

\section{Clonogenicity and asymmetric division assays}

Single cells were plated in 96-well plates and incubated with $1 \% \mathrm{O}_{2}$ or $21 \% \mathrm{O}_{2}$ to observe sphere formation at 3, 7, 14 and 21 days. The newly formed spheres were cultured in stem cell medium and differentiation medium to observe asymmetric division at 1, 3 and 5 days.

\section{Protein detection}

Briefly, proteins and mRNAs were detected in GBM tissues and GBM and U87MG cells cultured in $21 \% \mathrm{O}_{2}$ or
$1 \% \mathrm{O}_{2}$ using immunofluorescence staining, western blotting, RT-qPCR, ELISA and immunohistochemistry and the detailed methods are presented in the supplementary materials.

\section{Flow cytometry (FCM) analysis}

FCM was used to analyse the cell cycle of GBM cells cultured in $21 \% \mathrm{O}_{2}$ or $1 \% \mathrm{O}_{2}$. In addition, cells were exposed to TMZ $(400 \mu \mathrm{M})$ and cultured in $1 \% \mathrm{O}_{2}$ for another $72 \mathrm{~h}$ to detect apoptosis, and the detailed methods are presented in the supplementary materials.

\section{$\mathrm{LDH}$ release assay}

Cells at a density of $5 \times 10^{4}$ in a $100-\mu l$ suspension were seeded in 96-well plates and cultured in the presence of TMZ in $21 \% \mathrm{O}_{2}$ or $1 \% \mathrm{O}_{2}$ for $72 \mathrm{~h}$, and $\mathrm{LDH}$ release was detected with a LDH assay kit according to the manufacturer's instructions. A detailed description of the method is presented in the Supplementary methods.

\section{CCK-8 assay}

GBM and U87MG cells were cultured in 96-well plates in $21 \% \mathrm{O}_{2}$ or $1 \% \mathrm{O}_{2}$ for $72 \mathrm{~h}$ and in the absence or presence of TMZ $(400 \mu \mathrm{M})$ for another $48 \mathrm{~h}$ to detect cell proliferation. The IC50 values were also calculated by performing CCK-8 assays, and the detailed methods are presented in the supplementary materials.

\section{Prediction of the HIF1a and HIF2a binding sites in EGF}

Hypothetical HIF $1 \alpha$ and HIF $2 \alpha$ binding sites in EGF promoter were predicted using http://jaspar.genereg.net/. In addition, EGF promoter activity was measured by comparing the luciferase levels. A detailed description of the method used to detect promoter activity is presented in the supplementary materials.

\section{HIF knockout assays}

HIF-knockout (HIF-KO) cells were prepared with HIF1 $\alpha$ and HIF2 $\alpha$ sgRNAs, and the detailed methods are described in the supplementary materials.

\section{miRNA-Seq analysis}

Control, HIF $1 \alpha-\mathrm{KO}$, HIF $2 \alpha-\mathrm{KO}$ and HIF $1 \alpha / \mathrm{HIF} 2 \alpha-\mathrm{KO}$ cells were cultured under hypoxic conditions for $24 \mathrm{~h}$ and then collected for the miRNA-Seq analysis. A detailed description of the method is presented in the supplementary materials and the results were uploaded in the NCBI Gene Expression Omnibus (GEO) database (www.ncbi. nlm.nih.gov/geo) under accession number GSE142719.

\section{In vivo experiments}

BALB/c-nu mice (male, 4 6 weeks) were used in this study. GBM cells $\left(8 \times 10^{4}\right)$ were injected into the brains of 5 mice, and the animals were fed for 14 days. The tumours 
and normal tissues were collected to analyse HIF1 $\alpha$ and HIF2 $\alpha$ expression. HIF-KO cells $\left(8 \times 10^{4}\right)$ were injected into the brains of 200 mice (simple size for each group was estimated by $\left.\left(\mu_{\alpha}+\mu_{\beta}\right)^{2} \times p_{0} \times\left(1-p_{0}\right) /\left(p-p_{0}\right)^{2}\right)$, and the groups included Con, Con+TMZ, HIF1 $\alpha-\mathrm{KO}$, HIF $1 \alpha-\mathrm{KO}$ + TMZ, HIF $2 \alpha-\mathrm{KO}, \mathrm{HIF} 2 \alpha-\mathrm{KO}+\mathrm{TMZ}, \mathrm{HIF} 1 \alpha / \mathrm{HIF} 2 \alpha-$ $\mathrm{KO}$, and HIF $1 \alpha / \mathrm{HIF} 2 \alpha-\mathrm{KO}+\mathrm{TMZ}$. MRI was used to detect tumour volume in five randomly selected mice by SPSS 19.0 on day 21. Tumour tissues were collected from another five mice and protein expression was analysed using IHC, RT-qPCR and western blotting, as described above. The remaining mice were used to record the survival time, and the dead mice were excluded after implantation in three day. The ethics committee of Southwest Hospital at Army Medical University approved all animal procedures.

\section{Statistical analysis}

SPSS 19.0 software was used for statistical analyses. Data are presented as means \pm standard deviations (SDs). Student's $t$ test was used to assess the significance of differences between the two groups, and one-way analysis of variance (one-way ANOVA) was performed to compare data from at least three groups. The log-rank test was used to analyse the (Overall Survival) OS or (Disease Free Survival) DFS. Pearson's correlation coefficients were calculated to analyse the correlations between genes. $P<0.05$ was considered a statistically significant difference.

\section{Results}

Effects of HIF1a/HIF2a on the survival time of patients with GBM

According to TCGA database, HIF1 $\alpha$ was expressed at higher levels in GBM tissues than in normal tissues, but a significant difference in HIF2 $\alpha$ expression was not observed between tumour and normal tissues (Fig. 1a-d). In addition, high HIF1 $\alpha$ expression led to a shorter DFS and OS, but the DFS and OS of patients were not related to HIF2 $\alpha$ expression (Fig. 1e-h). Culturing GBM1 cells in $1 \% \mathrm{O}_{2}$ for $6,12,24$ and $48 \mathrm{~h}$ increased HIF1 $\alpha$ expression. However, no significant difference in HIF2 $\alpha$ expression was observed during hypoxia (Fig. $1 \mathrm{i}$ and S1A, B). GBM1 cells were implanted into the brains of mice housed in $10 \% \mathrm{O}_{2}$ for 14 days. HIF1 $\alpha$ was expressed at higher levels in tumour tissues than in normal tissues, but a significant difference in HIF2 $\alpha$ expression was not observed (Fig. 1i and S1C). In addition, immunohistochemistry verified the effects of the hypoxic microenvironment on GBM tissues (Fig. 1i). Finally, RT-qPCR and western blotting revealed increasing levels of HIF1 $\alpha$ in World Health Organization (WHO) grade II glioma to grade IV tumours, but no difference in HIF2 $\alpha$ expression was observed (Fig. $1 \mathrm{j}$ and S1D).

\section{Hypoxia promoted arrest in $\mathrm{G} 1$ phase and inhibited cell apoptosis}

Hypoxyprobe $^{\mathrm{TM}}-1$ was used to verify that the cells were maintained in the hypoxic microenvironment (Fig. 2a). The hypoxic cells had a higher proliferation rate and a higher proportion of cells in $G_{1}$ phase than the normoxic cells (Fig. 2b, c and S2A). Then, the addition of TMZ (0, $100,200,400$ and $800 \mu \mathrm{M})$ into the medium of GBM cells resulted in lower levels of $\mathrm{LDH}$ release under hypoxic conditions (Fig. 2d and S2B). Additionally, the cells exposed to TMZ $(400 \mu \mathrm{M})$ for $72 \mathrm{~h}$ under normoxic conditions were presented higher percentages of later and total apoptosis compared with hypoxic cells (Fig. 2e and $\mathrm{S} 2 \mathrm{C})$. Finally, the IC50 value for GBM1 cells cultured under normoxic conditions was $845.10 \pm 423.82 \mu \mathrm{mol} / \mathrm{L}$, which was much lower than the value for cells cultured under hypoxic conditions $(1678.28 \pm 586.87 \mu \mathrm{mol} / \mathrm{L}$, Fig. 2f). A similar significant difference was observed in GBM2 cells (Fig. S2D).

\section{Hypoxia promoted the dedifferentiation of GBM cells}

Morphological changes were observed in only one cell exposed to $21 \% \mathrm{O}_{2}$ or $1 \% \mathrm{O}_{2}$, and the cell was dead after exposure to $21 \% \mathrm{O}_{2}$ for 21 days. However, the cells cultured with $1 \% \mathrm{O}_{2}$ formed suspended spheres after one week, and the rate of spheres (spheres/d3 surviving cells) increased in a time-dependent manner, with a value greater than $95 \%$ after exposure for 21 days (Fig. 2g-h). Next, differentiation was assessed in the newly formed spheres cultured with DMEM/F12 $+10 \%$ foetal bovine serum (FBS) under a state of growth adherence and the features of stemness were verified after an incubation with DMEM/F12 + EGF + FGF2 + B27 in a state of suspended growth (Fig. 2i). Immunofluorescence staining showed newly formed spheres and the cells cultured in $1 \% \mathrm{O}_{2}$ for $72 \mathrm{~h}$ expressed CD133, CD15, Nestin, ABCG2, HIF1 $\alpha$ and HIF2 $\alpha$ at high levels (Fig. 2j).

\section{Simultaneous HIF1a/HIF2a-KO promoted cell proliferation and chemosensitization}

Successful knockout of HIF1 $\alpha$ and HIF2 $\alpha$ was confirmed by immunofluorescence staining (Fig. S3A). The CCK-8 assay did not reveal significant differences in proliferation among HIF $1 \alpha-\mathrm{KO}$ cells, HIF $2 \alpha-\mathrm{KO}$ cells and the control group after culture under hypoxic conditions for $72 \mathrm{~h}$. However, the proliferation rate increased significantly after simultaneous HIF $1 \alpha$ and HIF $2 \alpha$ knockout (Fig. 3a, S3B and S5E). In addition, single knockout of HIF $1 \alpha$ or HIF2 $\alpha$ did not affect the number of cells in $G_{1}$ phase, but fewer cells with simultaneous HIF1 $\alpha$ and HIF $2 \alpha$ knockout were detected in $G_{1}$ phase (Fig. $3 b$ and S5E). Nevertheless, after TMZ exposure, cells with simultaneous HIF1 $\alpha$ and HIF2 $\alpha$ knockout showed the lowest proliferation rate, the highest level of LDH release, 


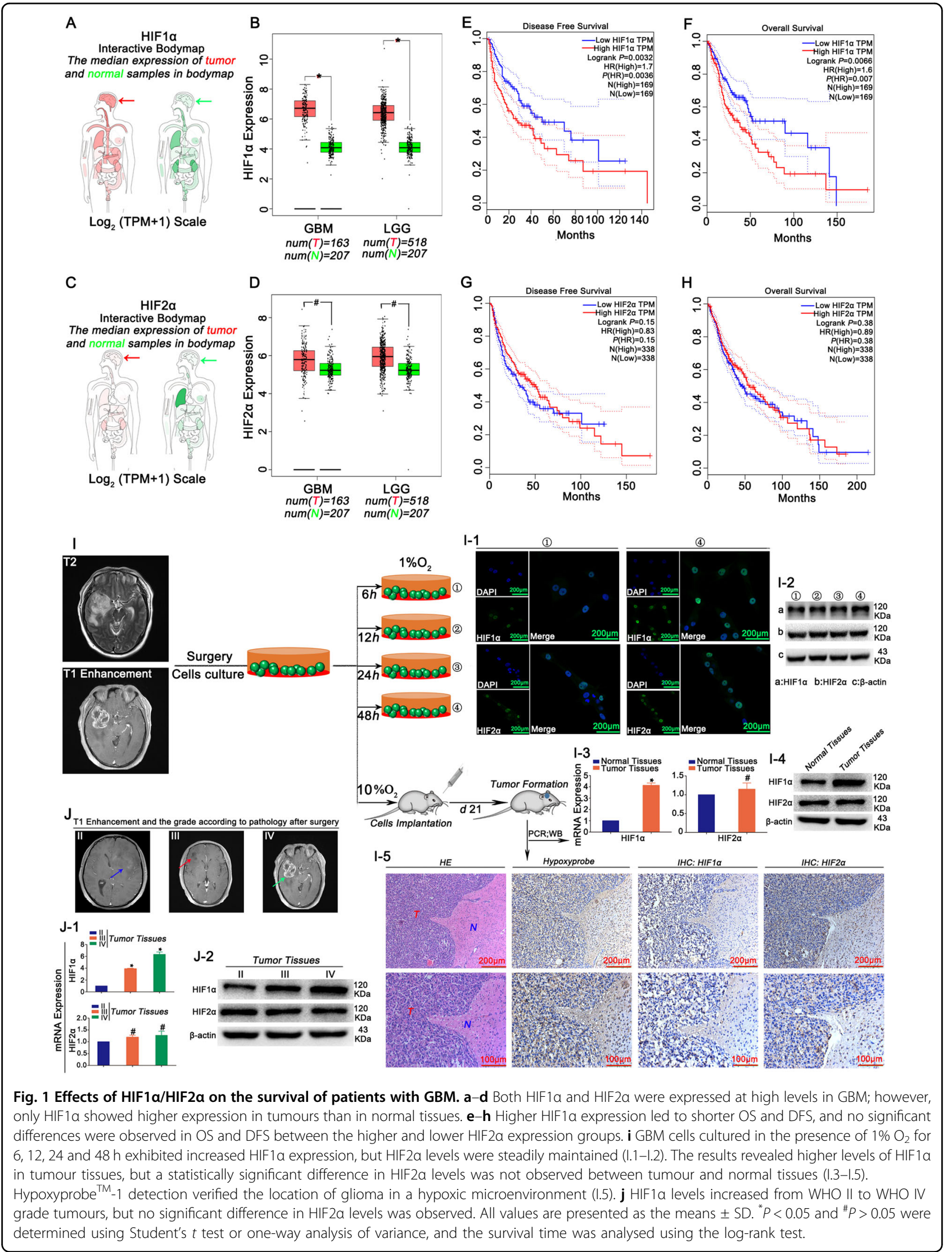




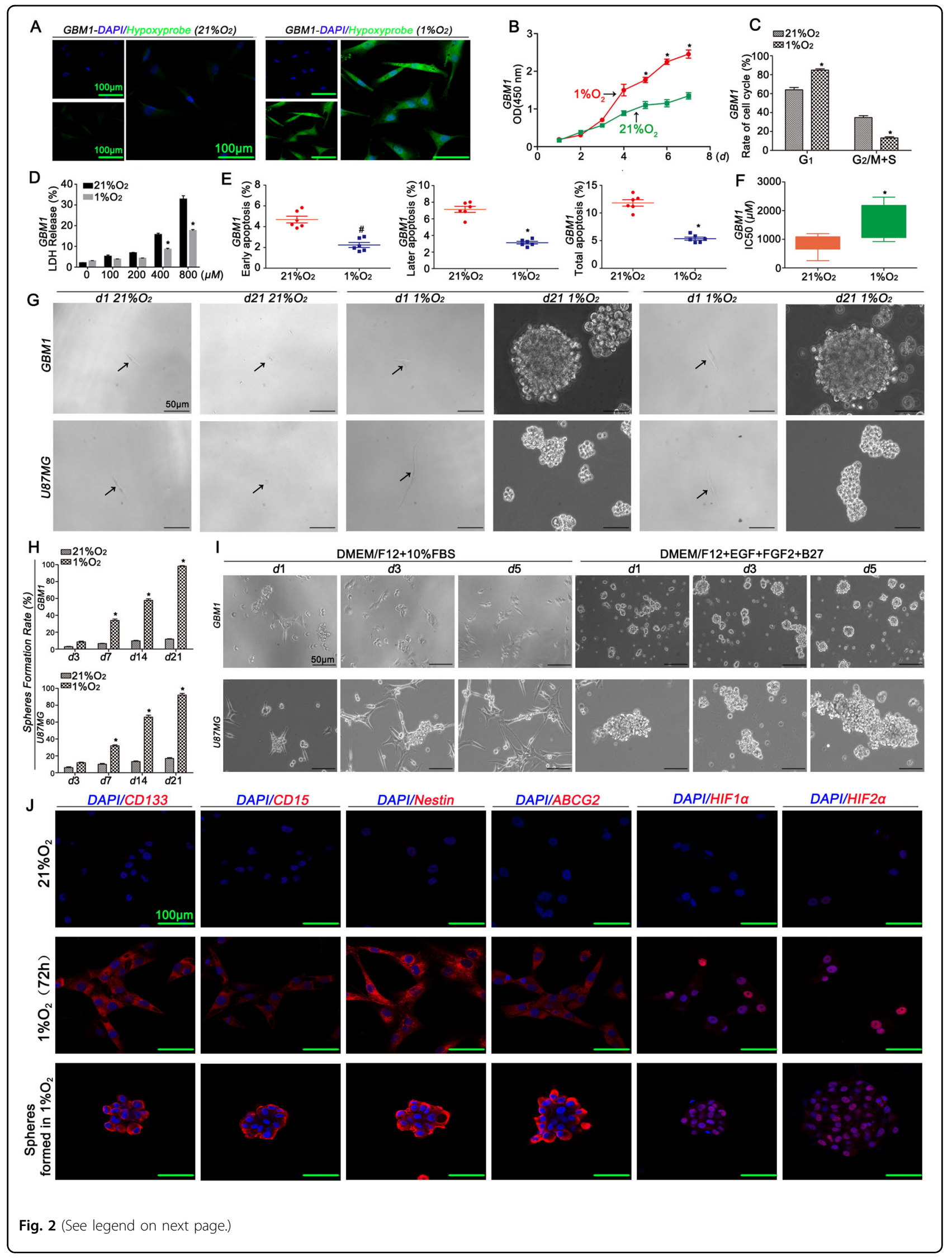


(see figure on previous page)

Fig. 2 Hypoxia inhibited apoptosis and induced the dedifferentiation of GBM cells. a GBM1 cells cultured in the presence of $1 \% \mathrm{O}_{2}$ presented higher levels of Hypoxyprobe ${ }^{T M}-1$. $\mathbf{b}$ GBM1 cells cultured in the presence of $1 \% \mathrm{O}_{2}$ displayed a higher proliferation rate than cells cultured in the presence of $21 \% \mathrm{O}_{2}$. c GBM1 cells exposed to hypoxia for $72 \mathrm{~h}$ displayed a higher proportion of cells in $\mathrm{G}_{1}$ phase. $\mathbf{d} \mathrm{TMZ}(0,100,200,400 \mathrm{and} 800 \mu \mathrm{MM})$ was added to the culture medium of GBM1 cells, and lower levels of LDH release were observed in the hypoxia group than in the control group. e TMZ $(400 \mu \mathrm{M})$ was added to the culture medium of cells cultured in the presence of different concentrations of oxygen for $72 \mathrm{~h}$, and lower percentages of late and total apoptotic cells were observed in the GBM1 cells cultured with $1 \% \mathrm{O}_{2}$, but no difference was observed in the percentage of early apoptotic cells between the two groups. $\mathbf{f} I \mathrm{C} 50$ values of GBM1 cells cultured under normoxic conditions were lower than cells cultured under hypoxic conditions. $\mathbf{g}-\mathbf{h}$ The sphere formation rate of cells cultured in the presence of $1 \% \mathrm{O}_{2}$ was higher than in cells cultured in the presence of $21 \% \mathrm{O}_{2}$. i Newly formed spheres exhibited asymmetric division. $\mathbf{j}$ Newly formed spheres and GBM1 cells cultured in the presence of $1 \% \mathrm{O}_{2}$ for $72 \mathrm{~h}$ expressed CD133, CD15, Nestin, ABCG2, HIF1a and HIF2a at high levels, which were not detected in cells cultured under normoxic conditions. ${ }^{*} P<$ 0.05 was determined using Student's $t$ test.

the highest apoptotic rate and the lowest IC50 value (Fig. 3c-f, S3C-F and S5E). The sphere formation rate by a single cell cultured in $1 \% \mathrm{O}_{2}$ decreased after HIF1 $\alpha$ or HIF $2 \alpha$ knockout, and the lowest value was observed after simultaneous HIF1 $\alpha$ and HIF2 $\alpha$ knockout (Fig. 3g, S4A and S5E). In addition, western blots showed an increase in HIF $1 \alpha$ levels after HIF2 $\alpha$ knockout, an increase in HIF2 $\alpha$ levels after HIF1 $\alpha$ knockout, and significantly decreased levels of CD133 and Nestin after HIF1 $\alpha$ and HIF2 $\alpha$ knockout (Fig. 3h, S4B and S5E). These cells were implanted into the brains of the mice, and cells with simultaneous HIF1 $\alpha$ and HIF2 $\alpha$ knockout produced tumours with a larger volume than control cells, and the tumours of both groups above were larger than groups implanted with single HIF1 $\alpha$ or HIF2 $\alpha$ knockout cells. An intraperitoneal injection of TMZ $(2 \mathrm{mg} / \mathrm{kg})$ into the aforementioned groups reduced the tumour volume in the HIF $1 \alpha$ or HIF $2 \alpha$ knockout groups, and the smallest tumour volume was observed in the group with dual HIF1 $\alpha$ and HIF $2 \alpha$ knockout (Fig. 3i. k, l, S5B-C and S5E). The tumour weight showed a similar trend (Fig. S5D, E). Regarding the survival time in the animals without TMZ exposure, HIF1 $\alpha$ or HIF $2 \alpha$ knockout alone correlated with a longer survival time than the control, but simultaneous HIF1 $\alpha$ and HIF2 $\alpha$ knockout correlated with a shorter survival time than the control. However, after TMZ exposure, the trend changed; the group with both HIF1 $\alpha$ and HIF2 $\alpha$ knockout showed the longest survival time compared with the other three groups (Fig. 3j, S5A and S5E).

\section{HIF $1 a$ and HIF2 $a$ expression were regulated by miR-210-3p under hypoxic conditions}

HIF1 $\alpha-\mathrm{KO}$, HIF2 $\alpha-\mathrm{KO}$, dual HIF1 $\alpha$ and HIF2 $\alpha$ knockout and control cells were subjected to miRNA-Seq analysis to identify miRNAs that target HIF1 $\alpha$ or HIF2 $\alpha$ (Fig. 4a). The results showed that only miR-210-3p was the common miRNA identified in the groups and presented a statistically significant relationship with HIF $1 \alpha$ and HIF2 $\alpha$ expression (Fig. 4b, c and S6A and Supplementary Table S1). The heat map and volcano plot revealed a decrease in miR-210-3p expression in HIF $1 \alpha-K O$ cells and an increase in its expression after
HIF2 $\alpha$ knockout. Next, we compared the group in which HIF1 $\alpha$ and HIF2 $\alpha$ were knocked out simultaneously with the control and found control group expressed miR-210-3p at higher levels. Finally, the HIF1 $\alpha-$ $\mathrm{KO}$ and HIF $2 \alpha-\mathrm{KO}$ groups were compared, and higher miR-210-3p expression was observed in the HIF $2 \alpha-K O$ group (Fig. 4b-d). RT-qPCR data verified this significant result (Fig. 4e and S6D). Glioma tissues were also detected, and the WHO IV group presented a higher level of miR-210-3p than the other groups (Fig. 4f), and the expression was much higher in tumour tissues than in normal tissues (Fig. 4g). Finally, according to TCGA database, the survival time was lower for patients with tumours displaying higher miR-210-3p expression (Fig. 4h). Next, the relationship between HIF1 $\alpha$, HIF2 $\alpha$ and miR-210-3p was examined. First, HIF $1 \alpha-K O$ or HIF2 $\alpha-K O$ GBM cells were cultured in $1 \% \mathrm{O}_{2}$, and miR210-3p was overexpressed or inhibited. HIF2 $\alpha$ levels decreased with miR-210-3p overexpression and increased when miR-210-3p expression was inhibited (Fig. 4i and S7). HIF1 $\alpha$ expression increased with miR-210-3p overexpression and decreased when miR-210-3p expression was inhibited (Fig. $4 \mathrm{j}$ and S7). Finally, the cell apoptosis assay showed that miR-210-3p overexpression in HIF1 $\alpha$ $\mathrm{KO}$ cells or miR-210-3p silencing in HIF $2 \alpha-\mathrm{KO}$ cells led to a higher apoptotic rate (Fig. 4k-1 and S6B-D).

\section{HIF1a and HIF2a regulated the malignant progression of GBM through EGF}

ELISA and immunofluorescence staining showed higher levels of EGF after cells were cultured in $1 \% \mathrm{O}_{2}$ for $72 \mathrm{~h}$ than in the normoxic cells, and the spheres formed in $1 \%$ $\mathrm{O}_{2}$ showed higher expression of EGF (Fig. 5a and S8A-B). Moreover, TCGA database showed high expression of EGF in GBM (Fig. 5b). The OS and DFS of patients with low EGF levels were much longer than patients with high EGF levels (Fig. 5c-d). TCGA and CCLE data showed that both HIF1 $\alpha$ and HIF2 $\alpha$ positively regulated EGF expression (Fig. 5e). The EGF promoter activity in the hypoxic group was higher than in the normoxic group, and lower EGF promoter activity was observed in the cells with single HIF $1 \alpha$ or HIF $2 \alpha$ knockout compared with the 


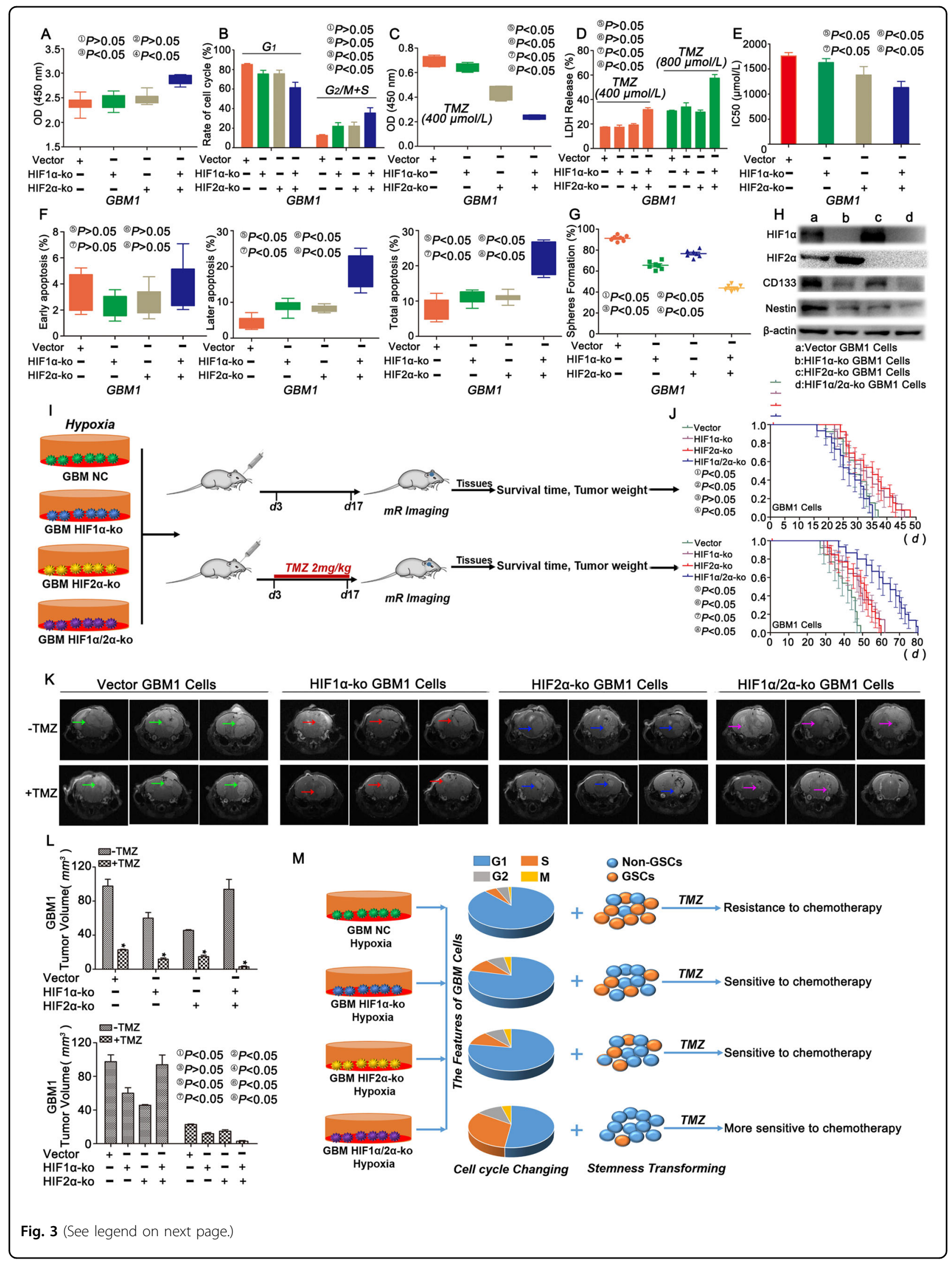


(see figure on previous page)

Fig. 3 Simultaneous HIF1a and HIF2a knockout increased proliferation and chemosensitization. a No significant difference in proliferation was observed in GBM1 cells with single HIF1a or HIF2a knockout, but a higher cell proliferation rate was observed in cells with simultaneous HIF1a and HIF2a knockout in the absence of the TMZ treatment. b GBM1 cells with single HIF1a or HIF2a knockout presented no significant differences compared with the control, but a significant difference was observed after simultaneous HIF1a or HIF2a knockout, as this group presented the lowest percentage of cells in $\mathrm{G}_{1}$ phase compared with the other groups cultured in the presence of $1 \% \mathrm{O}_{2}$. c HIF1a- or HIF2a-KO GBM1 cells exposed to TMZ $(400 \mu \mathrm{M})$ for $72 \mathrm{~h}$ showed decreased proliferation, and the lowest proliferation rate was observed after simultaneous HIF1a and HIF2a knockout. d Higher levels of LDH release were observed in simultaneous HIF1a- and HIF2a-KO cells than in other cells. e The IC50 value decreased significantly after HIF1a or HIF2a knockout. $\mathbf{f}$ The percentages of late and total apoptotic cells increased after HIF1a or HIF2a knockout, and the highest percentage of apoptotic cells was observed in the group with simultaneous HIF1a and HIF2a knockout, but no significant difference was observed in the percentage of early apoptotic cells among groups. g A lower sphere formation rate was observed after HIF1a or HIF2a knockout. $\mathbf{h}$ CD133 and Nestin expression decreased after HIF1a or HIF2a knockout in cells. HIF1a expression increased after HIF2a knockout. In contrast, HIF2a expression increased after HIF1a knockout. i Schematic of the in vivo assay. $\mathbf{j}-\mathbf{I}$ Analyses of the survival time and tumour volume in control and mice implanted with HIF1a/HIF2a-KO cells and treated with or without TMZ (2 mg/kg). $\mathbf{m}$ HIF1a or HIF2a knockout alone did not exert significant effects on proliferation and the cell cycle because of substitution effects, but inhibited stemness, leading to chemosensitization after TMZ treatment. However, if HIF1a and HIF2a were knocked out simultaneously, they inhibited cell cycle arrest, promoted proliferation, and decreased stemness, resulting in the chemosensitization of GBM cells. ${ }^{*} P<0.05$ was determined using Student's $t$ test, and the specific $P$ values are shown in Fig. S5E.

control cells. All the cells listed above exhibited higher EGF promoter activity than cells with dual HIF1 $\alpha$ and HIF $2 \alpha$ knockout (Fig. 5f, S8C and S8I). A similar trend was observed for mRNA and protein levels (Fig. 5g-h, S8D-E and S8I). In addition, the changes in sphere formation were recorded after the addition of EGF to the culture medium of HIF1 $\alpha$ - or HIF2 $\alpha-\mathrm{KO}$ cells, resulting an increase in the number of spheres (Fig. $5 \mathrm{i}$ and S8F-G). Finally, FCM revealed a decreased apoptotic rate after the addition of EGF to the culture medium of all groups (Fig. $5 \mathrm{j}$ and $\mathrm{S} 8 \mathrm{H}$ ). A bioinformatics analysis was performed to identify the hypoxia-response elements (HREs) in the EGF sequence, and two predicted binding regions were found: one sequence was $5^{\prime}$-AGGCGTGG- $3^{\prime}$ with a relative score of 0.889410 (site 1) and the other was $5^{\prime}$ GGGCGTGG- $3^{\prime}$ with a relative score of 0.913365 (site 2). The two predicted sequences were mutated and the change in luciferase activity in HIF $1 \alpha-$ or HIF $2 \alpha-K O$ cells was detected to verify the regulatory process (Fig. $5 \mathrm{k}$ ). For the control cells without HIF1 $\alpha$ or HIF $2 \alpha$ knockout, the luciferase activity decreased after each of the two predicted sequences was mutated and reached the lowest level in the group in which both sequences were mutated. For cells carrying site 1 or site 2 mutations alone, the results revealed decreased luciferase activity after HIF1 $\alpha$ or HIF $2 \alpha$ knockout, and the cells with simultaneous HIF1 $\alpha$ and HIF $2 \alpha$ knockout showed the lowest luciferase activity among groups. Then, the changes in the luciferase activity were analysed for cells after dual site 1 and site 2 mutations, and among all groups, the lowest luciferase activity was observed when HIF $1 \alpha$ and HIF $2 \alpha$ were simultaneously deleted (Fig. $5 \mathrm{l}$ and S8I).

\section{Discussion}

GBM is observed in the hypoxic microenvironment ${ }^{22,23}$, which is mainly regulated by HIF $1 \alpha$ and HIF $2 \alpha^{5,7,24}$. Previous studies explored the long-term contributions of these proteins to tumour growth and revealed an important role for HIF $1 \alpha^{2,8,25}$. HIF $2 \alpha$ primarily functions in glioma stem cells (GSCs) and promotes GSCs radiochemoresistance by maintaining stemness under hypoxia conditions $^{5,26}$. Therefore, the inhibition of HIF1 $\alpha$ or HIF $2 \alpha$ inhibits the malignant progression of GBM cells $^{8,25}$. For example, the inhibition of HIF1 $\alpha$ increases the sensitivity of GBM cells to chemotherapeutic drugs ${ }^{27}$ and a strategy targeting HIF2 $\alpha$ in GSCs attenuated the tumour initiation potential ${ }^{5,28}$. Therefore, these studies strongly support the necessary development of pharmacological HIF inhibitors as treatments for GBM, as they will theoretically inhibit tumour progression. As a result, several HIF inhibitors have been approved in phase $\operatorname{trials}^{29}$. However, until now no HIF-targeted therapies have cured patients successfully with GBM.

HIF $1 \alpha$ or HIF2 $\alpha$ was deleted to observe dedifferentiation, proliferation and chemoresistance in order to explore the failure of inhibitors targeting these proteins. Regarding dedifferentiation, previous studies have confirmed that GSCs develop from non-GSCs in response to therapeutic stress, such as $\mathrm{TMZ}^{30,31}$ and ionizing radia$\operatorname{tion}^{32}$. As a result, studies have proposed the hypothesis that hypoxia may also induce dedifferentiation ${ }^{33-35}$; however, none of the studies have conclusively verified this hypothesis. Therefore, we detected dedifferentiation and found more than $95 \%$ of single GBM cells formed spheres after culture under hypoxic conditions for 21 days, while very few spheres formed under normoxic conditions. Even if $20 \%$ of the newly formed spheres were derived from GSCs existing in GBM itself (the percentage of GSCs in GBM is approximately $20 \%{ }^{36}$ ), the other $75 \%$ of the newly formed spheres should be derived from differentiated non-GSCs after dedifferentiation. Our results confirming this mechanism were consistent with previous studies showing that stemness decreased after HIF1 $\alpha$ or HIF2 $\alpha$ knockout ${ }^{2,5,28}$. Nevertheless, unlike previous 

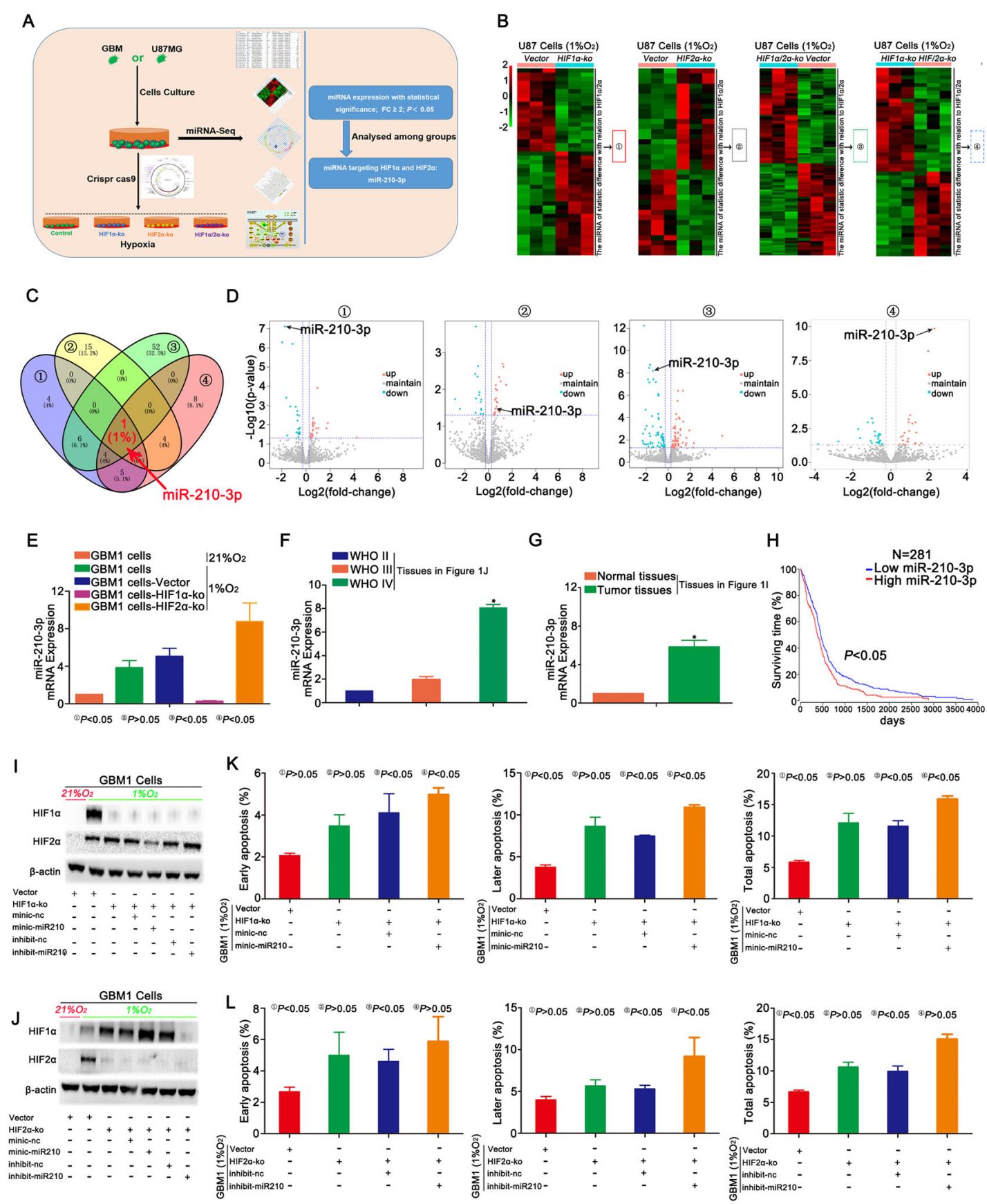

Fig. 4 miR-210-3p regulated HIF1 $a$ and HIF2a expression in hypoxic cells. a Schematic of the mechanistic study. A miRNA-Seq analysis of HIF1a$\mathrm{KO}, \mathrm{HIF} 2 \mathrm{a}-\mathrm{KO}$, simultaneous HIF1a- and HIF2a-KO and control cells was performed and revealed statistically significant differences in the expression of miRNAs targeting HIF1a or HIF2a in this process. $\mathbf{b}-\mathbf{d}$ Heat maps showed statistically significant changes in the expression of miR-210-3p associated with HIF1a and HIF2a expression. The expression of miR-210-3p decreased in HIF1a-KO cells compared with control cells; however, its expression increased after HIF2a knockout. Compared with the simultaneous HIF1a and HIF2a knockout group, the control group exhibited increased miR-210-3p expression. Finally, for the HIF1a-KO and HIF2a-KO groups, higher miR-210-3p expression was observed in the cells of the HIF2a-KO group. e The expression of miR-210-3p was detected in GBM1 cells after HIF1a and HIF2a knockout using RT-qPCR. $\mathbf{f}$ Higher miR-210-3p expression was observed in WHO grade IV tumours compared with other tumour grades. $\mathbf{g}$ Higher miR-210-3p expression was observed in tumour tissues than in normal tissues. $\mathbf{h}$ TCGA database showed a lower survival time in the group with higher miR-210-3p expression. $\mathbf{i}$, $\mathbf{j}$ Changes in HIF1a and HIF2a expression were detected in HIF1a- or HIF2a-KO GBM1 cells overexpressing or silencing for miR-210-3p and cultured in the presence of 1\% $\mathrm{O}_{2}$. $\mathbf{k}$, I Apoptosis was detected in HIF1a- or HIF2a-KO GBM1 cells overexpressing or silencing for miR-210-3p expression and cultured in the presence of $1 \% \mathrm{O}_{2} .{ }^{*} P<0.05$ was determined using Student's $t$ test, and the specific $P$ values are shown in Fig. S6D. 

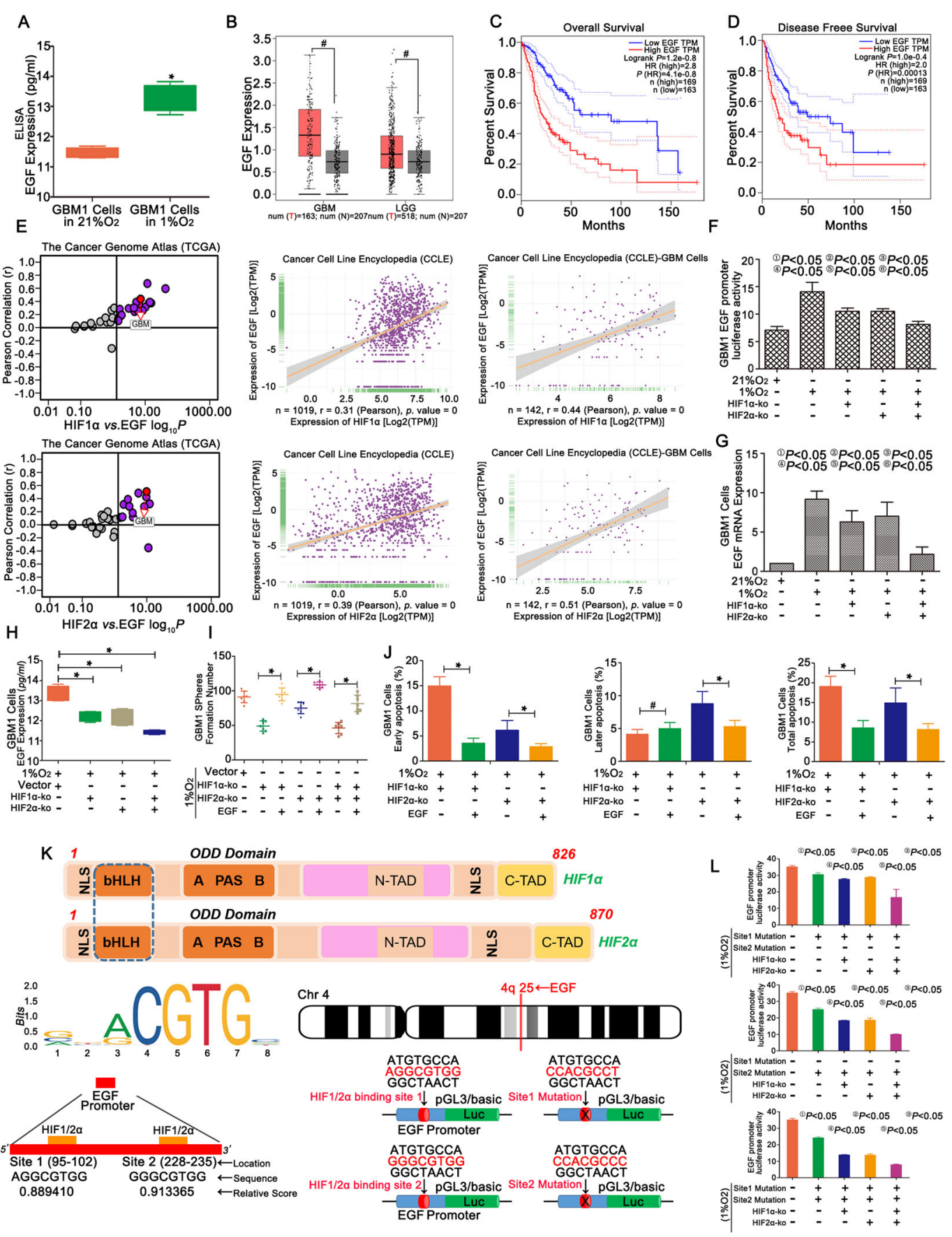

Fig. 5 HIF1a and HIF2a regulated GBM growth and apoptosis through EGF. a An ELISA showed higher levels of EGF in GBM1 cells after culture with $1 \% \mathrm{O}_{2}$ for $72 \mathrm{~h}$. b EGF was expressed at high levels in tumours, but the difference between the tumour and normal tissues was not significant. c, $\mathbf{d}$ Longer OS and DFS were observed in the group with lower EGF expression than in the control group. e A positive correlation was observed between HIF1a, HIF2a and EGF expression, according to TCGA and CCLE databases. $\mathbf{f}-\mathbf{h}$ Decreases in the luciferase activity of the EGF promoter and levels of the EGF mRNA and protein were observed in single HIF1a- or HIF2a-KO cells compared with the control. However, all of these groups showed higher levels than simultaneous HIF1a- and HIF2a-KO cells. $\mathbf{i}$ The number of spheres increased after EGF was added to the culture medium of HIF1 $a-$ or HIF2a-KO cells cultured in the presence of $1 \% \mathrm{O}_{2}$. $\mathbf{j}$ The percentage of apoptotic in HIF1 $\mathbf{a}$ - or HIF2a-KO cells decreased after the addition of EGF to the culture medium. $\mathbf{k}$ Bioinformatics analysis of the HREs of EGF based on EGF family binding sites. Two predicted binding regions were shared: one sequence was 5'-AGGCGTGG-3' and the other was 5'-GGGCGTGG-3'. In addition, the two predicted sequences were mutated. I Detection of the luciferase activity of the EGF promoter after the mutation of the two predicted sequences in control cells, HIF1a- or HIF2a-KO cells cultured in the presence of $1 \% \mathrm{O}_{2} \cdot{ }^{*} P<0.05$ and ${ }^{\#} P>0.05$ were determined using Student's $t$ test or one-way analysis of variance, and the specific $P$ values are shown in Fig. S8l. 


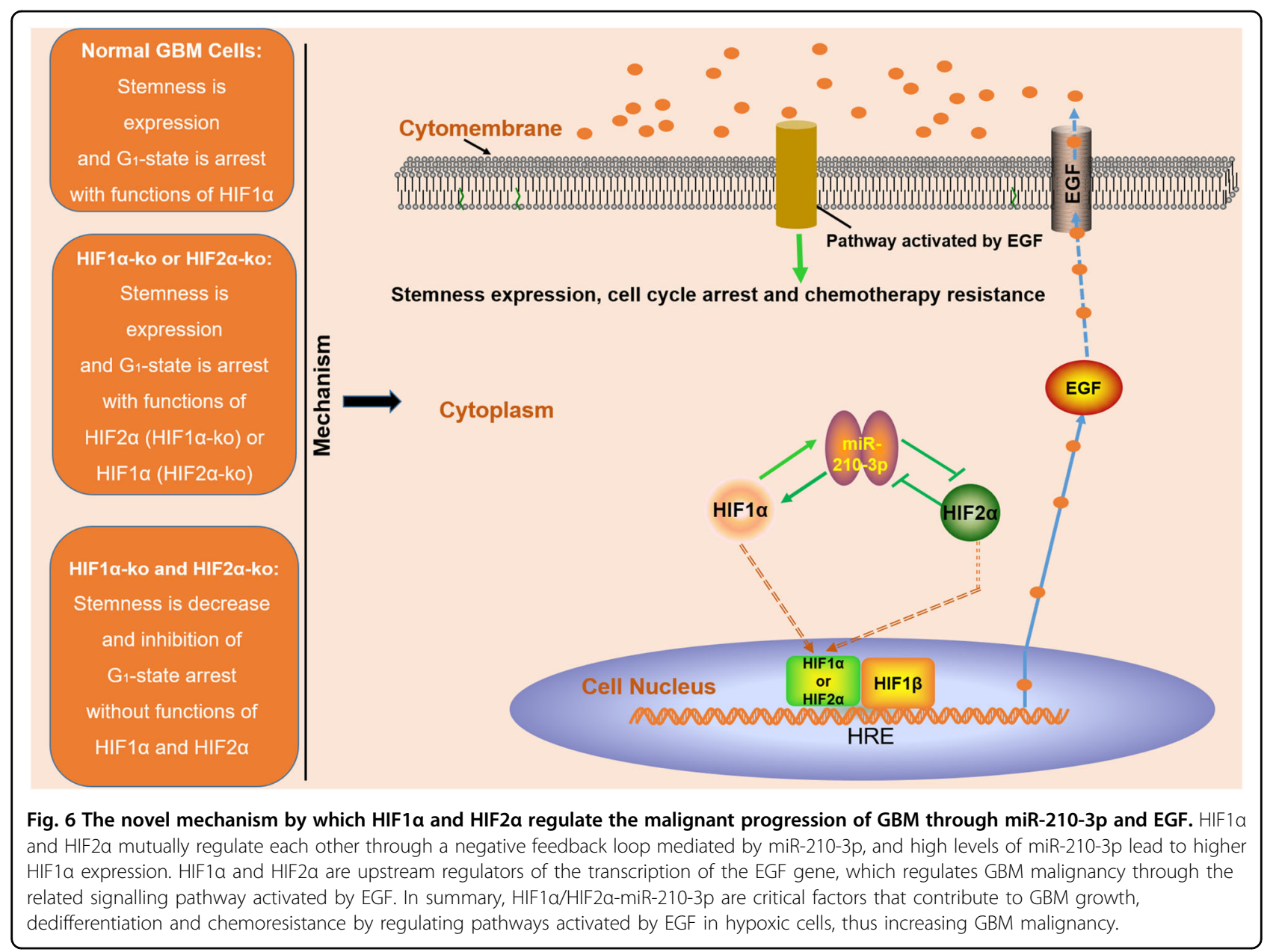

studies $^{37,38}$, no significant differences in proliferation and the cell cycle were observed in vitro after single HIF1 $\alpha$ or HIF2 $\alpha$ knockout, which may be due to the shorter detection time than used in previous studies. However, HIF $1 \alpha$ or HIF $2 \alpha$ knockout alone inhibited tumour growth in vivo. Furthermore, we emphasized that the proliferation rate was accelerated after simultaneous HIF $1 \alpha$ and HIF2 $\alpha$ knockout. This result revealed a substantial difference from the theory that simultaneous HIF $1 \alpha$ and HIF $2 \alpha$ knockout would result in the lowest proliferation rate. Surprisingly, the opposite result was observed after exposure to TMZ, indicating that simultaneous HIF1 $\alpha$ and HIF $2 \alpha$ knockout cells became chemosensitized. This study is significance, as GBM was successfully cured by simultaneously targeting both HIF $1 \alpha$ and HIF $2 \alpha$ and administering TMZ.

Next, we wondered why HIF $1 \alpha$ or HIF2 $\alpha$ knockout alone was unable to substantially decrease the tumour volume. Interestingly, the expression of HIF $2 \alpha$ increased after HIF $1 \alpha$ knockout and HIF1 $\alpha$ expression increased after HIF $2 \alpha$ knockout. Thus, another HIF $\alpha$ factor promotes tumour growth after one subunit is knocked out, leading to an unremarkable decrease in tumour volume. Consistent with previous studies, stemness was decreased after single HIF1 $\alpha$ or HIF $2 \alpha$ knockout, explaining why the knockout of either HIF1 $\alpha$ or HIF $2 \alpha$ resulted in chemosensitization. Nevertheless, simultaneously knockout of HIF $1 \alpha$ and HIF $2 \alpha$ not only promoted cell cycle progression without any substitution effects but also decreased the stemness more noticeably, remarkably increasing proliferation and chemosensitization (Fig. 3m). The mechanism by which HIF $1 \alpha$ and HIF $2 \alpha$ regulate each other remains unknown, and thus hypoxia-related miRNAs were the focus of this study. Both previous studies and our study indicated that a positive feedback loop existed between HIF1 $\alpha$ and miR-210-3 $\mathrm{p}^{17,39}$; however, no studies have elucidated the regulatory mechanism between HIF2 $\alpha$ and miR-210-3p. By performing experiments, we confirmed that high levels of HIF $1 \alpha$ increased miR-210-3p expression in hypoxic cells, restricting the expression of HIF $2 \alpha$. However, if HIF $1 \alpha$ was knocked out, miR-210-3p expression decreased, thus HIF $2 \alpha$ expression increased (Fig. 6). This new regulatory mechanism explains the relationship between HIF1 $\alpha, \operatorname{HIF} 2 \alpha$ and 
miR-210-3p, potentially representing a new target for GBM treatment.

EGF, an upstream protein of HIF $1 \alpha$, has been reported in glioma ${ }^{29,39}$. Unexpectedly, in the present study, EGF expression decreased after HIF1 $\alpha$ and HIF2 $\alpha$ knockout. Actually, the two HIF $\alpha$ proteins are highly homologous and regulate some similar genes, suggesting that they may bind to similar HRE sequences ${ }^{40,41}$. Both HIF1 $\alpha$ and HIF $2 \alpha$ contain a conserved DNA binding region known as the bHLH-PAS domain, which is a common domain shared by HIF $1 \alpha$ and HIF2 $\alpha$ that binds to HREs and induces a series of responses ${ }^{8}$. Therefore, by performing a series of experiments, both HIF $1 \alpha$ and HIF $2 \alpha$ were verified as upstream genes that regulate EGF by binding the DNA sequences AGGCGTGG and GGGCGTGG in GBM cells. Therefore, feedback regulation exists in hypoxic cells between HIF1 $\alpha$, HIF2 $\alpha$ and EGF. EGF contributes to HIF1 $\alpha$ expression; in contrast, upregulation of HIF1 $\alpha$ and HIF $2 \alpha$ promoting EGF expression (Fig. 6).

In summary, researchers should create a better treatment to improve the prognosis of patients with GBM. Based on our findings, HIF $1 \alpha / H I F 2 \alpha-m i R-210-3 p$ regulates the malignant progression of GBM through EGF, which provides a new target strategy for GBM treatment.

\section{Acknowledgements}

The National Natural Science Foundation of China (NSFC 81802510 and 81672493) and the Key Laboratory of Tumour Immunology of the Ministry of Education (2018sjz103) financially supported this study.

\section{Author details}

${ }^{1}$ Department of Neurosurgery, Chongqing General Hospital, University of Chinese Academy of Sciences, 401147 Chongqing, China. ${ }^{2}$ Chongqing Medical University, 400016 Chongqing, China. ${ }^{3}$ Department of Oncology, Chongqing University Cancer Hospital, 400030 Chongqing, China. ${ }^{4}$ Chongqing Institute of Green and Intelligent Technology, Chinese Academy of Sciences, 400714 Chongqing, China. ${ }^{5}$ University of Chinese Academy of Sciences, 100049 Beijing, China

\section{Conflict of interest}

The authors declare that they have no conflict of interest.

\section{Publisher's note}

Springer Nature remains neutral with regard to jurisdictional claims in published maps and institutional affiliations.

Supplementary Information accompanies this paper at (https://doi.org/ 10.1038/s41419-020-03150-0).

Received: 22 May 2020 Revised: 14 October 2020 Accepted: 16 October 2020

Published online: 18 November 2020

\section{References}

1. Gagner, J. P., Lechpammer, M. \& Zagzag, D. Induction and assessment of hypoxia in glioblastoma cells in vitro. Methods Mol. Biol. 1741, 111-123 (2018).

2. Wang, P. et al. HIF1a regulates single differentiated glioma cell dedifferentiation to stem-like cell phenotypes with high tumorigenic potential under hypoxia. Oncotarget 8, 28074-28092 (2017).
3. Renfrow, J. J. et al. P08.14 In situ detection of hypoxia inducible factor 2 alpha in malignant gliomas. Neuro-Oncol. 19, iii57-iii57 (2017).

4. Young, R. M. \& Simon, M. C. Untuning the tumor metabolic machine: HIF-a: pro- and antitumorigenic? Nat. Med. 18, 1024-1025 (2012).

5. Li, Z. et al. Hypoxia-inducible factors regulate tumorigenic capacity of glioma stem cells. Cancer Cell 15, 501-513 (2009).

6. Koh, M. Y., Lemos, R., Liu, X. \& Powis, G. The hypoxia-associated factor switches cells from HIF-1a- to HIF-2a-dependent signaling promoting stem cell characteristics, aggressive tumor growth and invasion. Cancer Res. 71, 4015-4027 (2011).

7. Johansson, E. et al. TMIC-46. tuning of hypoxic and pseudo-hypoxic glioma phenotypes in perivascular and perinecrotic niches. Neuro-Oncol. 19 vi253-vi253 (2017).

8. Keith, B., Johnson, R. S. \& Simon, M. C. HIF1a and HIF2a: sibling rivalry in hypoxic tumour growth and progression. Nat. Rev. Cancer 12, 9-22 (2011).

9. Man, J. et al. Hypoxic induction of vasorin regulates Notch1 turnover to maintain glioma stem-like cells. Cell Stem Cell 22, 104-118 (2017).

10. Wang, P. et al. HIF1a regulates glioma chemosensitivity through the transformation between differentiation and dedifferentiation in various oxygen levels. Sci. Rep. 7, 7965 (2017).

11. Wei, M. et al. Oroxylin A increases the sensitivity of temozolomide on glioma cells by hypoxia-inducible factor 1 a/hedgehog pathway under hypoxia. J. Cell. Physiol. 234, 17392-17404 (2019).

12. Abolghasemi, M. et al. MicroRNAs in breast cancer: Roles, functions, and mechanism of actions. J. Cell. Physiol. 235, 5008-5029 (2019).

13. Wu, S. G., Chang, T. H., Liu, Y. N. \& Shih, J. Y. MicroRNA in lung cancer metastasis. Cancers (Basel) 11, pii:E265 (2019).

14. Yue, X., Lan, F. \& Xia, T. Hypoxic glioma cell-secreted exosomal miR-301a activates $W n t / \beta$-catenin signaling and promotes radiation resistance by targeting TCEAL7. Mol. Ther. 27, 1939-1949 (2019).

15. $\mathrm{Hu}$, J. et al. MiR-215 is induced post-transcriptionally via HIF-drosha complex and mediates glioma-initiating cell adaptation to hypoxia by targeting KDM1B. Cancer Cell 29, 49-60 (2016).

16. Jana, A., Narula, P., Chugh, A. \& Kulshreshtha, R. Efficient delivery of anti-miR210 using Tachyplesin, a cell penetrating peptide, for glioblastoma treatment Int J. Pharm. 572, 118789 (2019).

17. Agrawal, R. et al. Hypoxic signature of microRNAs in glioblastoma: insights from small RNA deep sequencing. BMC Genomics 15, 686 (2014).

18. Cheng, J. C., Klausen, C. \& Leung, P. C. Hypoxia-inducible factor 1 alpha mediates epidermal growth factor-induced down-regulation of E-cadherin expression and cell invasion in human ovarian cancer cells. Cancer Lett. 329 197-206 (2013).

19. Secades, P., de Santa-María, I. S., Merlo, A., Suarez, C. \& Chiara, M. D. In vitro study of normoxic epidermal growth factor receptor-induced hypoxia-inducible factor-1-alpha, vascular endothelial growth factor, and BNIP3 expression in head and neck squamous cell carcinoma cell lines: Implications for antiepidermal growth factor receptor therapy. Head. Neck 37, 1150-1162 (2015).

20. Henze, A. T. et al. Loss of PHD3 allows tumours to overcome hypoxic growth inhibition and sustain proliferation through EGFR. Nat. Commun. 5, 5582 (2014).

21. Zheng, H. L. et al. Oligomer procyanidins (F2) repress HIF-1a expression in human U87 glioma cells by inhibiting the EGFR/ AKT/mTOR and MAPK/ERK1/ 2 signaling pathways in vitro and in vivo. Oncotarget 8, 85252-85262 (2017).

22. $\mathrm{Hu}, \mathrm{M}$. et al. Correlation of hypoxia as measured by fluorine-8 fluoroerythronitroimidazole (18F-FETNIM) PET/CT and overall survival in glioma patients. Eur. J. Nucl. Med Mol. Imaging 47, 1427-1434 (2020).

23. Wang, P., Wan, W.-W, Xiong, S.-L., Feng, H. \& Wu, N. Cancer stem-like cells can be induced through dedifferentiation under hypoxic conditions in glioma, hepatoma and lung cancer. Cell Death. Discovery 3, 16105 (2017).

24. Tang, J. H. et al. Downregulation of HIF-1a sensitizes U251 glioma cells to the temozolomide (TMZ) treatment. Exp. Cell Res. 343, 148-158 (2016).

25. Renfrow, J. J. et al. Hypoxia-inducible factor 2a: a novel target in gliomas. Future Med. Chem. 10, 2227-2236 (2018).

26. Lee, S. B. et al. An ID2-dependent mechanism for VHL inactivation in cancer. Nature 529, 172-177 (2016).

27. Chen, Z. et al. In vitro angiogenesis by human umbilical vein endothelial cells (HUVEC) induced by three-dimensional co-culture with glioblastoma cells. J. Neurooncol. 92, 121-128 (2009).

28. Bhagat, M. et al. HIF-2a mediates a marked increase in migration and stemness characteristics in a subset of Glioma cells under hypoxia by activating an Oct-4/Sox-2- Mena (INV) axis. Int. J. Biochem. Cell Biol. 74, 60-71 (2016). 
29. Womeldorff, M., Gillespie, D. \& Jensen, R. L. Hypoxia-inducible factor-1 and associated upstream and downstream proteins in the pathophysiology and management of glioblastoma. Neurosurgical Focus 37, E8 (2014).

30. Lee, G. et al. Dedifferentiation of glioma cells to glioma stem-like cells by therapeutic stress-induced HIF signaling in the recurrent GBM model. Mol. Cancer Ther. 15, 3064-3076 (2016).

31. Auffinger, B. et al. Conversion of differentiated cancer cells into cancer stemlike cells in a glioblastoma model after primary chemotherapy. Cell Death Differ. 21, 1119-1131 (2014).

32. Dahan, P. et al. lonizing radiations sustain glioblastoma cell dedifferentiation to a stem-like phenotype through survivin: possible involvement in radioresistance. Cell death Dis. 5, e1543 (2014).

33. Li, P., Zhou, C., Xu, L. \& Xiao, H. Hypoxia enhances stemness of cancer stem cells in glioblastoma: an in vitro study. Int. J. Med. Sci. 10, 399-407 (2013).

34. Bar, E. E., Lin, A., Mahairaki, V., Matsui, W. \& Eberhart, C. G. Hypoxia increases the expression of stem-cell markers and promotes clonogenicity in glioblastoma neurospheres. Am. J. Pathol. 177, 1491-1502 (2010).
35. Mathieu, J. et al. HIF induces human embryonic stem cell markers in cancer cells. Cancer Res. 71, 4640-4652 (2011).

36. Singh, S. K. et al. Identification of human brain tumour initiating cells. Nature 432, 396-401 (2004).

37. Denko, N. C. Hypoxia, HIF1 and glucose metabolism in the solid tumour. Nat. Rev. Cancer 8, 705-713 (2008).

38. Kumar, H. \& Choi, D. K. Hypoxia inducible factor pathway and physiological adaptation: a cell survival pathway? Mediators Inflamm. 2015, 584758 (2015).

39. Li, J. et al. ELTD1 facilitates glioma proliferation, migration and invasion by activating JAK/STAT3/HIF-1 a signaling axis. Sci. Rep. 9, 13904 (2019).

40. Samanta, D., Gilkes, D. M., Chaturvedi, P., Xiang, L. \& Semenza, G. L. Hypoxiainducible factors are required for chemotherapy resistance of breast cancer stem cells. Proc. Natl Acad. Sci. USA 111, E5429-E5438 (2014).

41. Brahimi-Horn, M. C. \& Pouysségur, J. HIF at a glance. J. Cell. Sci. 122, 1055-1057 (2009). 